INPLASY

PROTOCOL

To cite: Zhao et al. Association between Greenspace and Blood Pressure: A Systematic Review and Meta-Analysis. Inplasy protocol 2021100033. doi:

10.37766/inplasy2021.10.0033

Received: 10 October 2021

Published: 10 October 2021

Corresponding author:

YaJun Chen

chenyj68@mail.sysu.edu.cn

Author Affiliation:

School of Public Health, Sun

Yat-sen University.

Support: NSFC81673139.

Review Stage at time of this submission: Completed but not published.

Conflicts of interest: None declared.

\section{Association between Greenspace and Blood Pressure: A Systematic Review and Meta-Analysis}

Zhao, Y1; Bao, W2; Yang, B3; Liang, J4; Gui, Z5; Huang, S6; Chen, Y7; Dong, G8; Chen, Y9.

Review question / Objective: Hypertension is a major risk factor for cardiovascular disease, which remains the leading cause of mortality worldwide. Urban environment as an possible cause of hypertension has received great attention. We aimed to systematically review the associations between greenspace and blood pressure (BP) levels/hypertension and use a meta-analysis to quantify the effects of greenspace on BP levels/hypertension.

Condition being studied: Many epidemiological studies have assessed associations of greenspace with blood pressure levels and/or the prevalence of hypertension, whereas the results was inconsistent with some studies showing positive association but the others reporting null or negative associations. Systematic review and meta-analysis was conducted to synthesize data quantitatively across studies based on the primary outcome.

INPLASY registration number: This protocol was registered with the International Platform of Registered Systematic Review and Meta-Analysis Protocols (INPLASY) on 10 October 2021 and was last updated on 10 October 2021 (registration number INPLASY2021100033).

\section{INTRODUCTION}

Review question / Objective: Hypertension is a major risk factor for cardiovascular disease, which remains the leading cause of mortality worldwide. Urban environment as an possible cause of hypertension has received great attention. We aimed to systematically review the associations between greenspace and blood pressure (BP) levels/hypertension and use a metaanalysis to quantify the effects of greenspace on BP levels/hypertension.

Condition being studied: Many epidemiological studies have assessed 
associations of greenspace with blood pressure levels and/or the prevalence of hypertension, whereas the results was inconsistent with some studies showing positive association but the others reporting null or negative associations. Systematic review and meta-analysis was conducted to synthesize data quantitatively across studies based on the primary outcome.

\section{METHODS}

Search strategy: We systematically searched Web of Science, PubMed, and Embase for all of the studies concerning greenspace and BP levels/hypertension published up to 25 April 2021. The search terms included indexed terms from "Emtree" in Embase, "MeSH" in PubMed, and title/abstract terms. Our search strategies used combinations of keywords concerning surrounding greenspace (e.g., greenness, green spaces, green area and greenery, etc.) and hypertension (e.g., blood pressure, high blood pressure, hypertensive and systolic/diastolic blood pressure, etc.). The search was restricted to the English language.

Participant or population: General human population of all ages, no restrictions on participants.

Intervention: Assessed greenspace exposure using an objective measure (including normalized difference vegetation index (NDVI), proportion of greenspace, distance to greenspace), or subjective measures (including self-reported frequency of visits to greenspaces, quality of green space).

Comparator: Exposed to higher greenspace compare with those exposed to lower levels.

Study designs to be included: Human epidemiological studies involving cohort, case-control, and cross-sectional designs.

Eligibility criteria: According to the PECOS (Population, Exposure, Comparator, Outcome, Study) statement, we included those studies if they met the following criteria (Morgan et al. 2018): (P) general human population of all ages, no restrictions on participants; (E) assessed greenspace exposure using an objective measure (including NDVI, proportion of greenspace, distance to greenspace), or subjective measures (including selfreported frequency of visits to greenspaces, quality of green space ); (C) exposed to higher greenspace compare with those exposed to lower levels; (0) definition of adult hypertension included SBP $\geq 140 \mathrm{mmHg}$ and/or DBP $\geq 90 \mathrm{mmHg}$, self-report of doctor-diagnosed hypertension, self-reportedly taking antihypertensive medicine, or the International Classification of Disease, 10th revision for hypertension (ICD10:I10) or ICD9:401; childhood hypertension was defined as average SBP and/or DBP that is $\geq 95$ th percentile for sex, age, and height (Falkner et al. 2004); (S) human epidemiological studies involving cohort, case-control, and cross-sectional designs. For duplicate publications that used data from the same study, we included the paper that reported the most comprehensive results.

Information sources: We systematically searched Web of Science, PubMed, and Embase for all of the studies concerning greenspace and BP levels/hypertension published up to 25 April 2021. The search terms included indexed terms from "Emtree" in Embase, "MeSH" in PubMed, and title/abstract terms. Our search strategies used combinations of keywords concerning surrounding greenspace (e.g., greenness, green spaces, green area and greenery, etc.) and hypertension (e.g., blood pressure, high blood pressure, hypertensive systolicblood pressure and diastolic blood pressure, etc.). The search was restricted to the English language. In addition, we manually searched the references lists of relevant reviews for potential articles.

Main outcome(s): The prevalence of hypertension and hypertension-related outcomes: systolic blood pressure and diastolic blood pressure. 
Quality assessment / Risk of bias analysis: Study quality was assessed using the Newcastle-Ottawa Scale (NOS) for cohort and case-control studies, and using the Practical Application of Clinical Evidence System (JBI PACES) (http: / / www.joannabriggs.edu.au/) for crosssectional studies. We also evaluated the risk of bias (ROB) for each of the selected studies using the U.S. National Institutes of Environmental Health Sciences National Toxicology Program Office of Health Assessment and Translation (OHAT). Further, we followed the Grading of Recommendations Assessment Development and Evaluation (GRADE) working group guidelines to evaluate the credibility of the pooled evidence.

Strategy of data synthesis: We categorized greenspace exposure into four groups: (1) NDVI (100m, 250m/300m, 500m, 1000m); (2) proportion of greenspace (percentage of green space or total green space around the residence); (3) distance to greenspace and (4) others (e.g., the quality of green space, the number of parks near participant's homes). We did not perform meta-analysis for the group of "others", but we summarized the results as part of our review. Meta-analysis was performed for a threshold number of 3 studies measuring the same exposure and outcome combination. We standardized effect estimates unit to a 0.1 increase in NDVI, per $1 \%$ increase in proportion of greenspace and per $500 \mathrm{~m}$ difference in distance to greenspace.

\section{Subgroup analysis: NA.}

Sensitivity analysis: We conducted the sensitivity analyses to estimate the stability of our results by excluding one study each time. Funnel plot and Egger's regression test were used to detect the potential publication bias. Additionally, the trim-fill method was used to test and adjust for publication bias.

Country(ies) involved: China.
Keywords: Blood pressure; Hypertension; Greenspace; Systematic review; Metaanalysis.

Contributions of each author:

Author 1 - Yu Zhao.

Email: zhaoy369@mail2.sysu.edu.cn

Author 2 - WenWen Bao.

Email: baoww3@mail2.sysu.edu.cn

Author 3 - BoYi Yang.

Email: yangby23@mail.sysu.edu.cn

Author 4 - JingHong Liang.

Email: liangjh78@mail2.sysu.edu.cn

Author 5 - ZhaoHuan Gui.

Email: guizhh@mail2.sysu.edu.cn

Author 6 - Shan Huang.

Email: huang0shan@126.com

Author 7 - YiCan Chen.

Email: chenyc55@mail2.sysu.edu.cn

Author 8 - GuangHui Dong.

Email: donggh5@mail.sysu.edu.cn

Author 9 - YaJun Chen.

Email: chenyj68@mail.sysu.edu.cn 